\title{
CS Research Square

\section{Long Non-Coding RNA ANRIL Promotes Doxorubicin Resistance in Triple-Negative Breast Cancer via Suppressing Glycolysis Through the MicroRNA-125a/ENO Pathway}

\author{
Jianli Ma \\ Harbin Medical University \\ Wenhui Zhao \\ Harbin Medical University \\ Han Zhang \\ Harbin Medical University \\ Zhong Chu \\ Harbin Medical University \\ Huili Liu \\ Harbin Medical University \\ Xue Fang \\ Harbin Medical University \\ Dabei Tang ( $\nabla$ tangdabeisy@hrbmu.edu.cn) \\ Harbin Medical University Cancer Hospital https://orcid.org/0000-0003-0994-3067
}

\section{Research Article}

Keywords: Long non-coding RNA, ANRIL, miR-125a, ENO, TNBC, chemotherapy

Posted Date: November 23rd, 2021

DOI: https://doi.org/10.21203/rs.3.rs-1062354/v1

License: @ (i) This work is licensed under a Creative Commons Attribution 4.0 International License. Read Full License 


\section{Abstract}

\section{Background}

Breast cancer is the main cause of death among women worldwide. More and more long non-coding RNAs (IncRNAs) have been identified as oncogenes or tumor suppressors during cancer development. However, whether ANRIL is involved in drug resistance in triple-negative breast cancer (TNBC) has not been investigated.

\section{Methods}

Luciferase reporter assay was conducted to verify the binding of miR-125a and ANRIL. RT-PCR and western blot were performed to detect the expression of miR-125a, ANRIL and ENO1. Gene silence and overexpression experiments as well as CCK-8 and colony formation assays on TNBC cell lines were performed to determine the regulation of molecular pathways. Glycolysis analysis was performed with Seahorse extracellular flux methodology.

\section{Results}

ANRIL expression in TNBC patients and TNBC cells was examined and we found that ANRIL expression was upregulated in both TNBC patients and TNBC cell lines. Knockdown of ANRIL increased the cytotoxic effect of ADR and inhibited HIF-1a-dependent glycolysis in TNBC cells. In addition, we found that ANRIL negatively regulated miR-125a expression in TNBC cell lines. Besides, a dual-luciferase reporter assay proved ANRIL functioned as a sponger of miR-125a. Further investigation revealed that ENO1 was a target of miR-125a and positively regulated by ANRIL in TNBC cells. Additionally, ANRIL upregulation reversed miR-125-mediated inhibition on HIF-1a-dependent glycolysis in TNBC cells. More notably, 2deoxy-glucose (2-DG) attenuated ANRIL-induced increase of drug resistance in TNBC cells.

\section{Conclusions}

Taken together, our study was the first to identify that knockdown of ANRIL plays an active role in overcoming the drug resistance in TNBC by inhibiting glycolysis through the miR-125a/ENO1 pathway, which maybe serve useful for the development of novel therapeutic targets.

\section{Introduction}

Breast cancer is the main cause of death among women worldwide ${ }^{1,2}$. Triple-negative breast cancer (TNBC) which lacks the expression of HER2, progesterone, and estrogen receptors, possesses an aggressive clinical phenotype $\mathrm{e}^{3-5}$. Chemotherapy rather than a hormone or targeted immunotherapy is now used as standard treatment for TNBC patients ${ }^{6-8}$. Adriamycin/doxorubicin (ADR) is a cytotoxic drug (an anthracycline antibiotic) that is commonly used in chemotherapy in TNBC patients. Nevertheless, many TNBC patients develop resistance to ADR-therapy, which leads to relapse and patient death. 
Dysregulation of IncRNAs is frequently observed during carcinogenesis and contributes to cancer development ${ }^{9,10}$. Accumulating evidence suggests that IncRNAs are involved in the development of TNBC by regulating DNA damage repair, cell cycle, apoptosis and angiogenesis ${ }^{11-13}$. For example, IncRNA $\mathrm{HEIH}$ was shown to modulate cell proliferation and apoptosis through miR-4458/SOCS1 axis in TNBC ${ }^{14}$. LINC00096 increased cell proliferation by targeting miR-383-5p/RBM3 axis in TNBC ${ }^{15}$. LncRNA GAS5 was reported to promote apoptosis and inhibit proliferation of TNBC cells by targeting miR-196a-5p and miR-378a-5p/SUFU signalling ${ }^{16}$. LncRNA CCAT1 could promote TNBC cells migration and invasion by suppressing miR-218/ZFX signalling ${ }^{17}$. These studies evoke the potential of altering IncRNAs expression in future to represent a novel therapeutic approach to reverse anti-chemotherapy in TNBC patients. However, further studies and mechanistic investigations of the regulation mechanism of IncRNAsmediated drug resistance in TNBC are needed.

LncRNA ANRIL is an antisense non-coding RNA in the INK4A locus and is widespread in many types of human cancers such as breast cancer ${ }^{18} \llbracket$ ovarian cancer ${ }^{19,20}$, gastric cancer ${ }^{21}$, melanoma ${ }^{22,}{ }^{23}$ and nonsmall cell lung carcinoma ${ }^{24,25}$. In nasopharyngeal carcinoma cells, downregulation of IncRNA ANRIL inhibits proliferation, induces apoptosis, and enhances radiosensitivity in nasopharyngeal carcinoma cells through regulating miR-125a ${ }^{26}$. In TNBC tissue and cells, LncRNA ANRIL promoted carcinogenesis via sponging miR-199a in TNBC tumor pathological ${ }^{27}$. However, the role and the regulation mechanism of IncRNA ANRIL-mediated chemoresistance in TNBC remains unclear.

LncRNAs regulate gene expression via functioning as microRNA spong $\mathrm{e}^{28,29}$. miRNAs are short, noncoding RNA molecules that bind to 3'UTR of mRNAs to regulate target gene expressions. Many miRNAs are closely related to TNBC tumor progression and chemoresistance ${ }^{30,31}$. miR-125a has been reported to be abnormally expressed in prostate carcinoma ${ }^{32}$. Various studies have shown miR-125a to be closely related to tumor cell growth, differentiation, and metastasis ${ }^{33-35}$. Especially, miR-125a has been reported to control the proliferation, apoptosis, migration in MCF-7 breast cancer cells ${ }^{36}$. Nevertheless, whether and how miR-125a regulates drug resistance in TNBC is largely unknown.

In this study, we provide compelling evidence that ANRIL was upregulated in TNBC patient tumor tissues and TNBC cell lines. Mechanistically, we discovered an ANRIL/miR-125a/ENO1 axis in mediating chemotherapy sensitivity against adriamycin. Our findings may provide insights into conquering chemotherapy resistance of TNBC patients.

\section{Methods}

Cell culture

The TNBC cell line MCF10A, MCF7, T47D, MDA-MB-231, BT549, and HS578T cells were kindly provided by the Cell Bank / Stem the Cell Bank, Chinese Academy of Sciences. The cells were cultured in 
Dulbecco's Modified Eagle's Medium (DMEM; ThermoFisher) containing 10\% fetal bovine serum (FBS; ThermoFisher), $1 \%$ penicillin, and streptomycin, and cultured at $37^{\circ} \mathrm{C}, 5 \% \mathrm{CO}_{2}$.

Total RNA isolation and qRT-PCR assays

RNA was extracted from the OS cellular samples using with TRIzol reagent (Thermo, USA). cDNA was generated by High-Capacity cDNA Reverse Transcription Kit (ThermoFisher). qRT-PCR was performed using the VII7 system (Applied Biosystems) with the SYBR ${ }^{\text {TM }}$ Green PCR Master Mix plus (ThermoFisher) for quantification analysis. RIP experiments were performed with the Magna RIP RNA-binding protein immunoprecipitation kit (Millipore, Billerica, MA, USA) according to the manufacturer's instructions. Coprecipitated RNAs were subjected to RT-qPCR analysis. U1 RNA was used as the housekeeping control. The primers used in this study are as follows: ANRIL-F: 5'-GCCTCATTCTGATTCAACA-3'; ANRIL-R: 5'TAGAAAGCAGTACTGACTCGG-3'.

Lentivirus vector construction and siRNA transfection

The knockdown and overexpression lentivirus vectors for ANRIL were produced by OBiO (Shanghai, China). The siRNAs against ANRIL (si-ANRIL) and negative control (NC) were synthesized by OBiO (Shanghai, China). TNBC cells were transfected with siRNAs with the Lipofectamine 3000 Reagent (Thermo, USA) according to the manufacturer's protocol.

Protein extraction and western blot analysis

Total proteins from the breast cancer tissues or the TNBC cells were extracted and analyzed as previously reported. The antibodies for the western blot analysis in this study were as follows: anti-HIF1a (Abcam, ab82832), anti-ENO1 (Abcam, ab85086), anti- $\beta$-actin (CST, \#3700).

Cell viability assay and colony formation assay

Cell Counting Kit-8 (CCK8) assay (Abcam, ab228554) was used for the Cell viability assessment according to the manufacturer's protocol. For the colony formation assay, cells were seeded in 12-well plates and then treated with ADR. The cells were harvested using trypsin, collected, and reseeded at a density of 2000 cells/well in 12-well plates 2 hours later. Then, the colonies were fixed and stained with $0.1 \%$ crystal violet 2 weeks later.

Cell apoptosis assay

Cell apoptosis was measured using an Annexin-V-FITC/PI double staining apoptosis kit (Abcam) according to the manufacturer's instructions. Flow cytometry (Beckman Coulter, CA, USA) was used to analyze apoptosis. Annexin- $\mathrm{V}^{+} / \mathrm{Pl}^{-}$cells were the apoptotic cells.

Xenograft tumor 
All live mice manipulations were performed according to guidelines and protocol approved by the Institutional Animal Care and Use Committee at Harbin Medical University Cancer Hospital. For xenograft experiments, BT-549 cells transfected with shANRIL or negative control vector were harvested and suspended in a density of $1 \times 10^{7}$ cells $/ 100 \mu \mathrm{L}$ in saline and injected subcutaneously into the right flank of each mouse. The volume of tumor was measured every 5 days and determined according to the equation: $0.5 \times$ length $\times$ width $^{2}$. At 4 -week post injection, the mice were killed by cervical dislocation, and the tumors were excised and photographed.

Immunohistochemistry

The tumor tissues were embedded with OCT and quickly frozen in the liquid nitrogen after weighting. 5$\mu$ m-thick sections were made in a Cryostat (Leica, Germany). Slides were blocked with $5 \%$ BSA at RT for 1 hour. The first antibody against Ki67 was incubated at RT for 2 hours a $37^{\circ} \mathrm{C}$. The second antibody was incubated for 1 hour at RT, and then uses diaminobenzidine (DAB) as chromogen.

Statistical analysis

The results are shown as the mean \pm SEM. The differences between the 2 groups were analyzed by a twotailed unpaired Student's t-test. One-way ANOVA followed by the Tukey multiple-comparisons test was applied for paired comparisons. Values of $p<0.05$ were considered statistically significant.

\section{Results}

ANRIL overexpression augmented Adriamycin (ADR)-induced cytotoxic effect in TNBC cells. To observe the expression change of ANRIL in TNBC, qRT-PCR was performed to detect its expression levels in 21 TNBC tissues and 10 normal breast tissues. We found a significantly higher level of ANRIL in TNBC tumor tissues (Figure 1A). Consistently, the expression level of ANRIL is also significantly higher in TNBC cell lines (MDA-MB-231, BT549, and HS578T) in comparison with ER + breast cancer cell lines (MCF7 and T47D) and normal breast cell line (MCF10 A) (Figure 1B). This analysis of ANRIL expression suggested that ANRIL might involve in TNBC progression. Due to the higher expression level of ANRIL in MDA-MB231 and BT549 cells, these 2 cell lines were transfected with si-ANRIL or si-NC and Vector or pcDNAANRIL to further investigate the role of ANRIL during drug resistance of TNBC cells in vitro. ANRIL expression was strikingly decreased in MDA-MB-231 and BT549 cells after transfection with si-ANRIL (Figure 1C). CCK8 assay implicated that cell viability was inhibited in MDA-MB-231 and BT549 cells treated with ADR, while si-ANRIL remarkably increased ADR-induced inhibition on cell viability (Figure 1D, E). Meanwhile, ANRIL overexpressed cells showed an obvious increase in ANRIL expression compared to vector-introduced cells (Figure 1F). Reversely, the results in si-ANRIL-introduced MDA-MB-231 and BT549 cells, CCK8 assay showed that ANRIL overexpression led to a marked increase in cell viability in ADRtreated MDA-MB-231 and BT549/ADR cells with respect to vector group (Figure 1G, H). Together, the above data revealed that ANRIL was related to the ADR resistance of TNBC cells. 
ANRIL knockdown inhibited HIF-1a-dependent glycolysis in TNBC and TNBC/ADR cells. More evidence demonstrated that increased glycolytic contributes to the development of drug resistance in different kinds of tumors ${ }^{37,38}$. Therefore, we investigated whether siANRIL negatively regulated aerobic glycolysis or not. We used the Seahorse Extracellular Flux Analyzer for glycolysis and mitochondrial respiration measurements, and ECAR to reflect aerobic glycolysis and OCR to indicate mitochondrial respiration. ANRIL knockdown in TNBC/ADR cells decreased glycolysis and glycolytic capacity, which was reflected in ECAR measurement (Figure 2A and 2B). Mitochondrial respiration was impaired in the process of aerobic glycolysis, resulting in a reduction of oxygen consumption that was reflected by OCR measurement. We observed that ANRIL knockdown enhanced OCR, indicating the positive roles of ANRIL in mitochondrial respiration (Figure 2C and 2D). Transcription factor hypoxia-inducible factor $1 \mathrm{a}(\mathrm{HIF-1a})$ controls the expression of a large number of gene products involved in energy metabolism and glycolysis. Therefore, we next investigated whether ANRIL knockdown mediated regulation of glycolysis was HIF-1a dependent. WB analysis indicated that knockdown of ANRIL effectively decreased HIF-1a in TNBC cells (Figure 2E and 2F). Therefore, these results demonstrated that ANRIL downregulation suppressed HIF-1a-dependent glycolysis.

ANRIL directly recognizes miR-125a in TNBC/ADR cells. The subcellular location of ANRIL determined by qRT-PCR showed that ANRIL was mainly inside the cytoplasm, indicating that ANRIL could contact with miRNAs in the cytoplasm (Figure 3A and 3B). We used microRNA.org, and miRBase to analyze the potential targets of ANRIL. The results provided the potential binding sequence of miR-125a to ANRIL, as shown in Figure 3C. Luciferase reporter assay further confirmed that miR-125a mimics but not miR-125a inhibitors reduced the luciferase activities of ANRIL-wt reporter vector in TNBC cells, but had no obvious inhibitory effect on ANRIL-mut reporter vector (Figure 3D and 3E). Anti-Ago2 RIP assay and luciferase reporter assay was conducted to further investigate whether ANRIL function as a sponger of miR-125a. The results showed that ANRIL and miR-125a have highly existed in Ago2-containing beads in TNBC cells relative to control IgG immunoprecipitates (Figure 3F). These results demonstrated that ANRIL is a sponge of miR-125a in TNBC cells.

ENO1 is a target of miR-125a and is positively regulated by ANRIL in TNBC cells. Bioinformatics analysis using targeting algorithms (TargetScan and microRNA.org) were used to further investigate the target protein of miR-125a. We found that 3'-UTR of ENO1 mRNA is a binding site of miR-125a (Figure 4A). Further luciferase reporter assay demonstrated that miR-125a upregulation significantly decreased the luciferase activity of ENO1-wt but not ENO1-mut in TNBC cells (Figure 4B). We next conducted qRT-PCR and western blot to see whether ANRIL knockdown or miR-125a overexpression could reduce ENO1 expression in TNBC cells. We found that both ANRIL silencing and miR-125a overexpression decreased the ENO1 expression both in mRNA level and protein level (Figure 4C and 4D). These results revealed that ANRIL increased ENO1 expression by functioning as a sponge of miR-125a in TNBC cells.

ANRIL increases TNBC cell chemoresistance via ENO1 in vitro. Except boosting tumorigenesis, the aerobic glycolysis of cancer cells provides an environment in which drug resistance is often increased. We used siRNA of ENO1 and 2-DG to see whether ENO1 was important for ANRIL during drug resistance 
in TNBC cells. The effect of ANRIL on TNBC cell drug resistance against ADR was determined by clone formation assay. We found that ANRIL overexpression significantly increased the clone formation ability of TNBC cells under ADR treatment (Figure 5A). Additionally, cell viability assay indicated that ANRIL promoted ADR resistance of TNBC cells (Figure 5B). Flow cytometry analysis also demonstrated that ANRIL-overexpression decreased apoptosis of TNBC cells treated with ADR. EN01knockdown by siRNA and 2-DG was used to treat ANRIL-overexpressing TNBC cells to investigate whether the TNBC cell resistance to ADR by ANRIL upregulation happens through increased glucose metabolism. The results revealed that drug resistance of ANRIL-upregulated TNBC cells to ADR was reversed by ENO1 SiRNA or 2DG (Figure 5).

ANRIL silencing inhibited tumor growth in vivo. To study the effect of ANRIL on TNBC ADR resistance in vivo, NOD-SCID mice were used for the xenograft tumor model. shANRIL cells were subcutaneously injected into the mammary fat pad of mice. To elucidate the role of ANRIL in chemoresistance in vivo model, ADR was administered locally in shNC- and shANRIL-treated mice. Macroscopic observation of the tumor tissue revealed that tumor volume was smaller in shANRIL mice than in the shNC mice under ADR treatment or not. Further measurement on tumor volume and weight results showed that both the tumor volume and weight in the shANRIL and ADR groups are smaller than the control group, while shANRIL+ADR group has smaller tumor volume and weight than the shANRIL-treated group, indicating that ANRIL suppression enhanced ADR sensitivity in TNBC (Figure 6A and 6B). We further evaluated whether ANRIL knockdown influences the apoptosis and proliferation ability of TNBC cells in chemoresistance in vivo model. We found that both shANRIL and ADR stimulation promoted the ratio of TUNEL positive cells and shANRIL+ADR has more TUNEL positive cells. Ki67 positive cells decreased in shANRIL and ADR groups and shANRIL+ADR has lesser Ki67 positive cells (Figure 6C and 6D). Taken together, our results suggested that ANRIL contributed to tumorigenesis of TNBC through regulating chemoresistance (Figure 7).

\section{Discussion}

In this study, we found that IncRNA ANRIL expression was upregulated in TNBC patients. ANRIL knockdown promoted the toxic effects of ADR and decreased HIF-1a-dependent glycolysis in TNBC cells. In TNBC cells, we discovered an ANRIL/miR-125a/ENO1 pathway in mediating chemotherapy sensitivity against ADR. Furthermore, 2-deoxy-glucose (2-DG) suppressed HIF-1a-dependent glycolysis and attenuated ANRIL-induced increase in ADR resistance in TNBC cells. We come to a conclusion that ANRIL plays a positive role in ADR resistance in TNBCby inhibiting glycolysis through the miR-125a/ENO1 pathway, contributing to a better understanding of the molecular mechanisms underlying TNBC chemoresistance.

Adriamycin has been widely used as a chemotherapy drug for the treatment of TNBC. However, TNBC patients often develop drug tolerance during chemotherapeutic treatment ${ }^{39}{ }^{40}$. Recent studies have shown that long non-coding RNA (IncRNA) may play an important regulatory role in tumor cell behavioral activities including invasion, proliferation, and drug resistance ${ }^{41,42}$. Jiang et al. found a novel IncRNA 
ARA plays an important role during adriamycin resistance in breast cancer. Our current findings have firstly found a relationship between IncRNA ANRIL and ADR resistance in TNBC. It was proved by experiments that down-regulation of ANRIL could promote ADR toxicity, while overexpression of ANRIL could partially promote $A D R$ resistance in $\mathrm{TNBC}^{43}$. In vivo experiments confirmed that knockdown of ANRIL reversed ADR resistance in TNBC cells xenograft in vivo. These results provided more evidence on the importance of IncRNA against drug-resistant TNBC.

Aerobic glycolysis has been raised been related to the development of drug-resistance $37,44,45$. The faster glucose metabolism is well recognized as one of the major hallmarks of malignancy and is related to tumor oncogenesis, progression, and metastasis. Besides, more and more evidence suggested that higher glycolysis may contribute to the development of drug resistance in multiple tumors, including TNBC. HIF1a plays a critical role in the regulation of glycolysis of cancer cells, has been demonstrated to be related to chemoresistance in many kinds of cancer cells ${ }^{46,47}$. We demonstrated in this study that downregulation of ANRIL decreased HIF-1a-dependent glycolysis. Except that, 2-DG treatment suppressed HIF-1a-dependent glycolysis and attenuated ANRIL-induced increase of drug-resistance in TNBC cells. Taken together, we conclude that ANRIL knockdown inhibited ADR-resistance by blocking HIF-1adependent glycolysis in TNBC cells.

In conclusion, our study shows for the first time that overexpression of ANRIL may lead to ADR resistance in TNBC, and downregulation of ANRIL partially reverses drug resistance. Moreover, down-regulation of ANRIL attenuated ADR resistance of TNBC by downregulating ENO1 expression. In vivo experiments confirmed that downregulation of ANRIL reversed ADR resistance in the TNBC xenograft mice model. Our results provided useful clues for exploring the mechanism of ADR resistance in TNBC, with certain application value and practical significance.

\section{Declarations}

\section{Availability of data and materials}

We hereby undertake that all data and materials are available.

\section{Acknowledgements}

None.

\section{Funding}

This study was funded by Youth Science Fund of National Natural Science Foundation of China (grant number: 81903063) and Haiyan Fund Project of Harbin Medical University Cancer Hospital.

Jianli Ma, Wenhui Zhao, Han Zhang, Zhong Chu, Huili Liu, Xue Fang, and Dabei Tang

\section{Authors' Contributions}


Conceptualization: JM and WZ; Data curation: DT; Formal analysis: HZ and ZC Funding acquisition: DT; Investigation: JM and DT; Methodology: HZ, ZC, HL and XF, Project administration: JM and WZ.

Supervision: DT; Roles/Writing-original draft: DT; Writing-review and editing: All authors. All authors read and approved the final manuscript.

\section{Ethics approval and consent to participate}

The study was approved by the Ethics Committee of Harbin Medical University Cancer Hospital.

\section{Consent for publication}

All listed authors have participated in the study and approved the submitted manuscript.

\section{Competing interests}

The authors declare that they have no conflict of interest.

\section{References}

1. Sun YS, Zhao Z, Yang ZN, Xu F, Lu HJ, Zhu ZY, Shi W, Jiang J, Yao PP and Zhu HP. Risk Factors and Preventions of Breast Cancer. International journal of biological sciences. 2017;13:1387-1397.

2. Waks AG and Winer EP. Breast Cancer Treatment: A Review. Jama. 2019;321:288-300.

3. Kumar P and Aggarwal R. An overview of triple-negative breast cancer. Archives of gynecology and obstetrics. 2016;293:247-69.

4. Sporikova Z, Koudelakova V, Trojanec R and Hajduch M. Genetic Markers in Triple-Negative Breast Cancer. Clinical breast cancer. 2018;18:e841-e850.

5. Medina MA, Oza G, Sharma A, Arriaga LG, Hernandez Hernandez JM, Rotello VM and Ramirez JT. Triple-Negative Breast Cancer: A Review of Conventional and Advanced Therapeutic Strategies. International journal of environmental research and public health. 2020;17.

6. De Laurentiis M, Cianniello D, Caputo R, Stanzione B, Arpino G, Cinieri S, Lorusso V and De Placido S. Treatment of triple negative breast cancer (TNBC): current options and future perspectives. Cancer treatment reviews. 2010;36 Suppl 3:S80-6.

7. Gupta GK, Collier AL, Lee D, Hoefer RA, Zheleva V, Siewertsz van Reesema LL, Tang-Tan AM, Guye ML, Chang DZ, Winston JS, Samli B, Jansen RJ, Petricoin EF, Goetz MP, Bear HD and Tang AH. Perspectives on Triple-Negative Breast Cancer: Current Treatment Strategies, Unmet Needs, and Potential Targets for Future Therapies. Cancers. 2020;12.

8. Jia H, Truica Cl, Wang B, Wang Y, Ren X, Harvey HA, Song J and Yang JM. Immunotherapy for triplenegative breast cancer: Existing challenges and exciting prospects. Drug resistance updates : reviews and commentaries in antimicrobial and anticancer chemotherapy. 2017;32:1-15.

9. Sanchez Calle A, Kawamura Y, Yamamoto Y, Takeshita F and Ochiya T. Emerging roles of long noncoding RNA in cancer. Cancer science. 2018;109:2093-2100. 
10. Lin YH. Crosstalk of IncRNA and Cellular Metabolism and Their Regulatory Mechanism in Cancer. International journal of molecular sciences. 2020;21.

11. Cao Q, Wu J, Wang X and Song C. Noncoding RNAs in Vascular Aging. Oxidative medicine and cellular longevity. 2020;2020:7914957.

12. Zhang $X$, Hong $R$, Chen $W, X u M$ and Wang $L$. The role of long noncoding RNA in major human disease. Bioorganic chemistry. 2019;92:103214.

13. Khawar MB, Mehmood R, Abbasi MH and Sheikh N. Multifactorial role of long non-coding RNAs (LncRNAs) in hematopoiesis. Frontiers in bioscience. 2018;10:119-126.

14. Li P, Zhou B, Lv Y and Qian Q. LncRNA HEIH regulates cell proliferation and apoptosis through miR4458/SOCS1 axis in triple-negative breast cancer. Human cell. 2019;32:522-528.

15. Tian Y, Xia S, Ma M and Zuo Y. LINC00096 Promotes the Proliferation and Invasion by Sponging miR-383-5p and Regulating RBM3 Expression in Triple-Negative Breast Cancer. OncoTargets and therapy. 2019;12:10569-10578.

16. Zheng S, Li M, Miao K and Xu H. IncRNA GAS5-promoted apoptosis in triple-negative breast cancer by targeting miR-378a-5p/SUFU signaling. Journal of cellular biochemistry. 2020;121:2225-2235.

17. Han C, Li X, Fan Q, Liu G and Yin J. CCAT1 promotes triple-negative breast cancer progression by suppressing miR-218/ZFX signaling. Aging. 2019;11:4858-4875.

18. Mehta-Mujoo PM, Cunliffe HE, Hung NA and Slatter TL. Long Non-coding RNA ANRIL in the Nucleus Associates With Periostin Expression in Breast Cancer. Frontiers in oncology. 2019;9:885.

19. Miao JT, Gao JH, Chen YQ, Chen H, Meng HY and Lou G. LncRNA ANRIL affects the sensitivity of ovarian cancer to cisplatin via regulation of let-7a/HMGA2 axis. Bioscience reports. 2019;39.

20. Qiu JJ, Lin YY, Ding JX, Feng WW, Jin HY and Hua KQ. Long non-coding RNA ANRIL predicts poor prognosis and promotes invasion/metastasis in serous ovarian cancer. International journal of oncology. 2015;46:2497-505.

21. Deng W, Zhang Y, Cai J, Zhang J, Liu X, Yin J, Bai Z, Yao H and Zhang Z. LncRNA-ANRIL promotes gastric cancer progression by enhancing NF-kB signaling. Experimental biology and medicine. 2019;244:953-959.

22. Sarkar D, Oghabian A, Bodiyabadu PK, Joseph WR, Leung EY, Finlay GJ, Baguley BC and AskarianAmiri ME. Multiple Isoforms of ANRIL in Melanoma Cells: Structural Complexity Suggests Variations in Processing. International journal of molecular sciences. 2017;18.

23. Xu S, Wang H, Pan H, Shi Y, Li T, Ge S, Jia R, Zhang H and Fan X. ANRIL IncRNA triggers efficient therapeutic efficacy by reprogramming the aberrant INK4-hub in melanoma. Cancer letters. 2016;381:41-8.

24. Xie Y, Zhang Y, Du L, Jiang X, Yan S, Duan W, Li J, Zhan Y, Wang L, Zhang S, Li S, Wang L, Xu S and Wang $\mathrm{C}$. Circulating long noncoding RNA act as potential novel biomarkers for diagnosis and prognosis of non-small cell lung cancer. Molecular oncology. 2018;12:648-658. 
25. Nie FQ, Sun M, Yang JS, Xie M, Xu TP, Xia R, Liu YW, Liu XH, Zhang EB, Lu KH and Shu YQ. Long noncoding RNA ANRIL promotes non-small cell lung cancer cell proliferation and inhibits apoptosis by silencing KLF2 and P21 expression. Molecular cancer therapeutics. 2015;14:268-77.

26. Hu $X$, Jiang $H$ and Jiang $X$. Downregulation of IncRNA ANRIL inhibits proliferation, induces apoptosis, and enhances radiosensitivity in nasopharyngeal carcinoma cells through regulating miR125a. Cancer biology \& therapy. 2017;18:331-338.

27. Xu ST, Xu JH, Zheng ZR, Zhao QQ, Zeng XS, Cheng SX, Liang YH and Hu QF. Long non-coding RNA ANRIL promotes carcinogenesis via sponging miR-199a in triple-negative breast cancer. Biomedicine \& pharmacotherapy $=$ Biomedecine $\&$ pharmacotherapie. 2017;96:14-21.

28. Kopp F and Mendell JT. Functional Classification and Experimental Dissection of Long Noncoding RNAs. Cell. 2018;172:393-407.

29. Zhao Z, Sun W, Guo Z, Zhang J, Yu H and Liu B. Mechanisms of IncRNA/microRNA interactions in angiogenesis. Life sciences. 2020;254:116900.

30. Piasecka D, Braun M, Kordek R, Sadej R and Romanska H. MicroRNAs in regulation of triple-negative breast cancer progression. Journal of cancer research and clinical oncology. 2018;144:1401-1411.

31. Kahraman M, Roske A, Laufer T, Fehlmann T, Backes C, Kern F, Kohlhaas J, Schrors H, Saiz A, Zabler C, Ludwig N, Fasching PA, Strick R, Rubner M, Beckmann MW, Meese E, Keller A and Schrauder MG. MicroRNA in diagnosis and therapy monitoring of early-stage triple-negative breast cancer. Scientific reports. 2018;8:11584.

32. Fu $\mathrm{Y}$ and Cao F. MicroRNA-125a-5p regulates cancer cell proliferation and migration through NAIF1 in prostate carcinoma. OncoTargets and therapy. 2015;8:3827-35.

33. Guo $X$, Wu Y and Hartley RS. MicroRNA-125a represses cell growth by targeting HuR in breast cancer. RNA biology. 2009;6:575-83.

34. Cai M, Chen Q, Shen J, Lv C and Cai L. Epigenetic silenced miR-125a-5p could be self-activated through targeting Suv39H1 in gastric cancer. Journal of cellular and molecular medicine. 2018;22:4721-4731.

35. Huang WK, Akcakaya P, Gangaev A, Lee L, Zeljic K, Hajeri P, Berglund E, Ghaderi M, Ahlen J, Branstrom R, Larsson C and Lui WO. miR-125a-5p regulation increases phosphorylation of FAK that contributes to imatinib resistance in gastrointestinal stromal tumors. Experimental cell research. 2018;371:287-296.

36. Liang Z, Pan Q, Zhang Z, Huang C, Yan Z, Zhang Y and Li J. MicroRNA125a5p controls the proliferation, apoptosis, migration and PTEN/MEK1/2/ERK1/2 signaling pathway in MCF7 breast cancer cells. Molecular medicine reports. 2019;20:4507-4514.

37. Desbats MA, Giacomini I, Prayer-Galetti T and Montopoli M. Metabolic Plasticity in Chemotherapy Resistance. Frontiers in oncology. 2020;10:281.

38. Ganapathy-Kanniappan S and Geschwind JF. Tumor glycolysis as a target for cancer therapy: progress and prospects. Molecular cancer. 2013;12:152. 
39. Wein $L$ and Loi S. Mechanisms of resistance of chemotherapy in early-stage triple negative breast cancer (TNBC). Breast. 2017;34 Suppl 1:S27-S30.

40. Nagini S. Breast Cancer: Current Molecular Therapeutic Targets and New Players. Anti-cancer agents in medicinal chemistry. 2017;17:152-163.

41. Qu Y, Tan HY, Chan YT, Jiang H, Wang N and Wang D. The functional role of long noncoding RNA in resistance to anticancer treatment. Therapeutic advances in medical oncology. 2020;12:1758835920927850.

42. Li Y, Yang $X$, Kang $X$ and Liu S. The regulatory roles of long noncoding RNAs in the biological behavior of pancreatic cancer. Saudi journal of gastroenterology : official journal of the Saudi Gastroenterology Association. 2019;25:145-151.

43. Jiang M, Huang O, Xie Z, Wu S, Zhang X, Shen A, Liu H, Chen X, Wu J, Lou Y, Mao Y, Sun K, Hu S, Geng $M$ and Shen K. A novel long non-coding RNA-ARA: adriamycin resistance-associated. Biochemical pharmacology. 2014;87:254-83.

44. Zong S, Dai W, Fang W, Guo X and Wang K. SIK2 Promotes Cisplatin Resistance Induced by Aerobic Glycolysis in Breast Cancer Cells through PI3K/AKT/mTOR Signaling Pathway. Bioscience reports. 2020.

45. Lorito N, Bacci M, Smiriglia A, Mannelli M, Parri M, Comito G, Ippolito L, Giannoni E, Bonechi M, Benelli M, Migliaccio I, Malorni L, Chiarugi P and Morandi A. Glucose Metabolic Reprogramming of ER Breast Cancer in Acquired Resistance to the CDK4/6 Inhibitor Palbociclib(.). Cel/s. 2020;9.

46. Griggio V, Vitale C, Todaro M, Riganti C, Kopecka J, Salvetti C, Bomben R, Bo MD, Magliulo D, Rossi D, Pozzato G, Bonello L, Marchetti M, Omede P, Kodipad AA, Laurenti L, Del Poeta G, Mauro FR, Bernardi R, Zenz T, Gattei V, Gaidano G, Foa R, Massaia M, Boccadoro M and Coscia M. HIF-1 alpha is overexpressed in leukemic cells from TP53-disrupted patients and is a promising therapeutic target in chronic lymphocytic leukemia. Haematologica. 2020;105:1042-1054.

47. Wang J, Yu Z, Wang J, Shen Y, Qiu J and Zhuang Z. LncRNA NUTM2A-AS1 positively modulates TET1 and HIF-1A to enhance gastric cancer tumorigenesis and drug resistance by sponging miR376a. Cancer medicine. 2020;9:9499-9510.

\section{Figures}


A

B
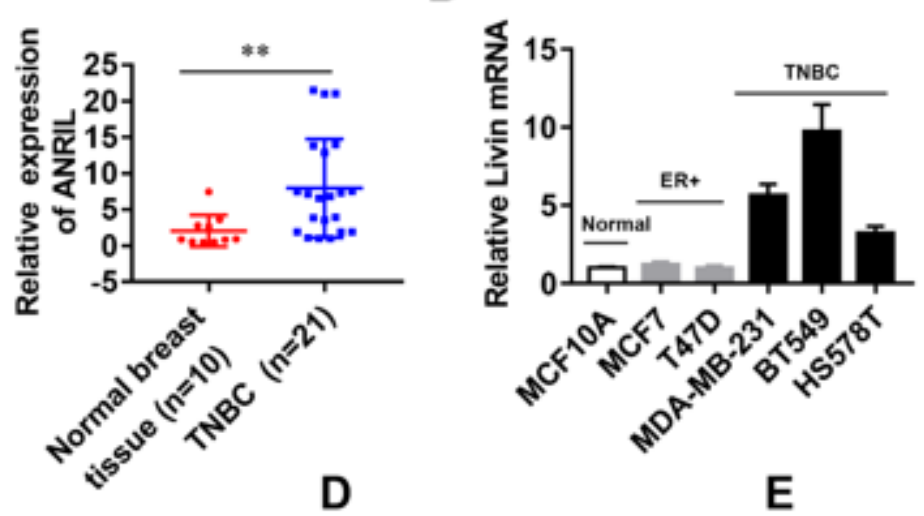

C
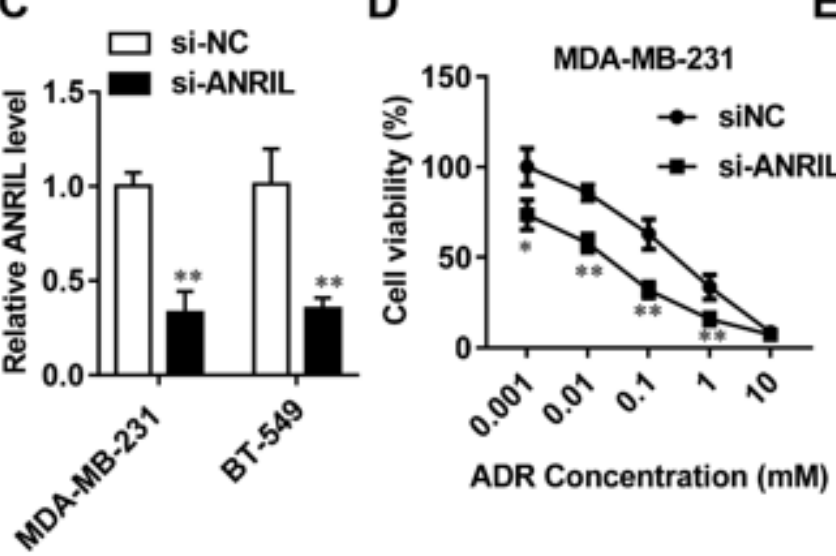

ADR Concentration (mM)
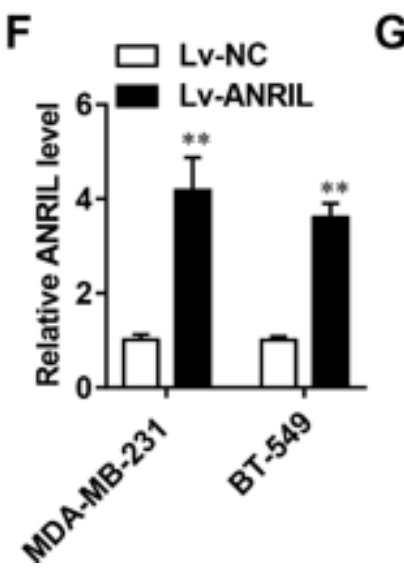

E

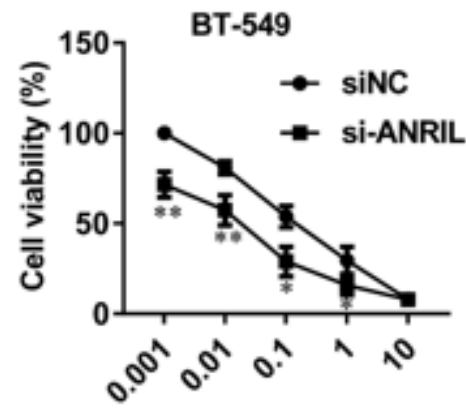

ADR Concentration (mM)

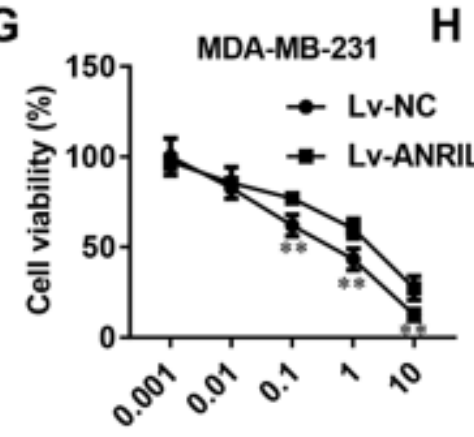

ADR Concentration (mM)

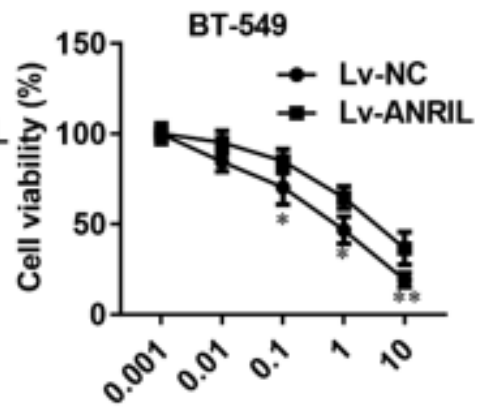

ADR Concentration (mM)

Figure 1

ANRIL overexpression augmented adriamycin-induced cytotoxic effect in TNBC cells. (A) The QRT-PCR analysis of ANRIL expression in TNBC tissues and normal breast tissue from different clinical samples. (B) The expression level of ANRIL in a panel of normal breast epithelial cell lines and breast cancer cell lines. (C) QRT-PCR analysis of ANRIL in primary TNBC cells transfected with ANRIL siRNA. (D, E) The cell viability of MDA-MB-231 and BT549 cells transfected with ANRIL siRNA were evaluated by CCK8 assay. (F) QRT-PCR analysis of ANRIL in primary TNBC cells infected with lentivirus carrying ANRIL sequence (Lv-ANRIL). (G, H) The cell viability of MDA-MB-231 and BT549 cells infected with Lv-ANRIL were evaluated by CCK8 assay. ${ }^{\star} p<0.05,{ }^{*} \mathrm{p}<0.01$. 
A

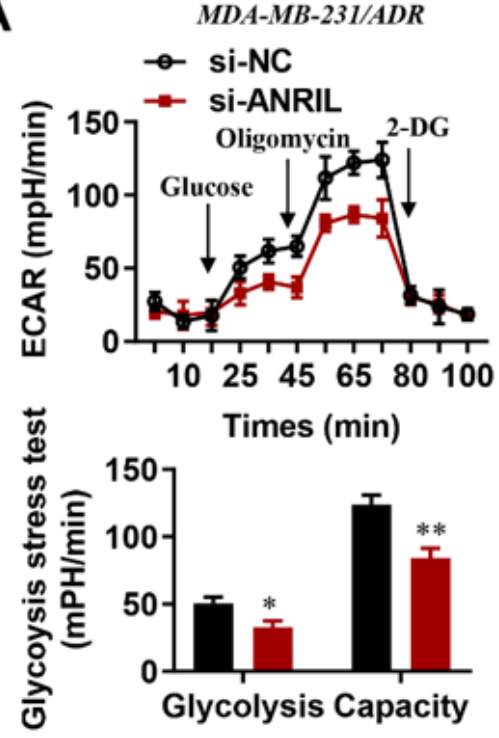

C
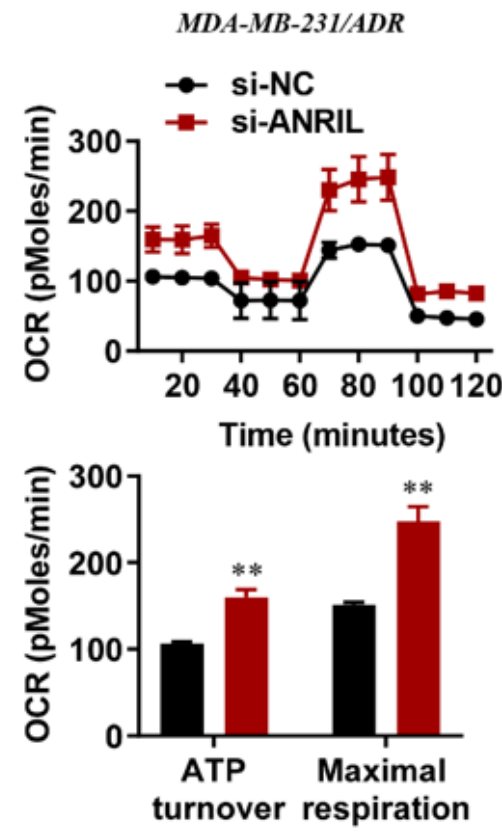

E

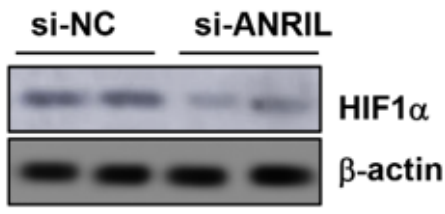

MDA-MB-231/ADR
B

BT-549/ADR

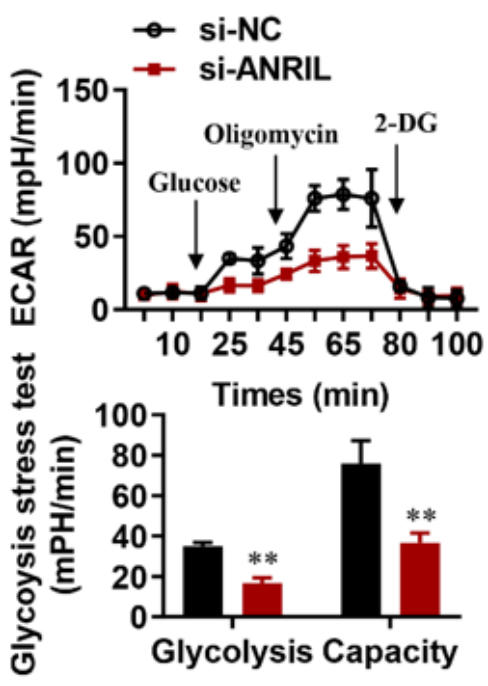

BT-549/ADR
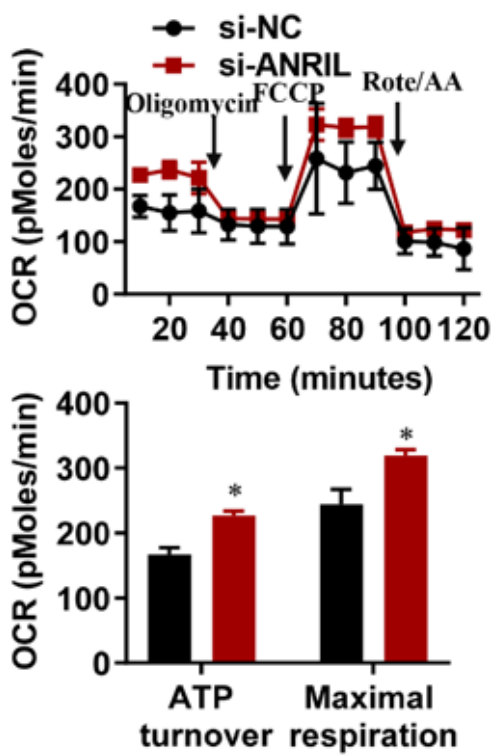

$\mathbf{F}$

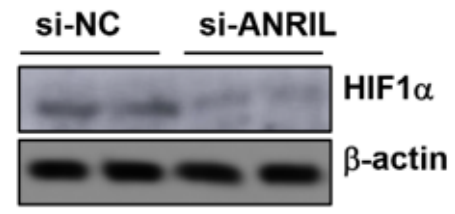

$B T-549 / A D R$

Figure 2

ANRIL knockdown inhibited HIF-1a-dependent glycolysis in TNBC and TNBC/ADR cells. (A, B) Diagram and quantitative of ECAR results obtained by Seahorse extracellular flux analyzer to determine the impact of ANRIL knockdown on aerobic glycolysis in MDA-MB-231 and BT549 cells. (C, D) Diagram and quantitative of OCR measurement with Seahorse analyzer to confirm the role of ANRIL knockdown in 
mitochondrial respiration. (E, F) WB analysis showed that knockdown of ANRIL effectively lowered HIF-1a level in MDA-MB-231 and BT549 cells treated by ADR. ${ }^{*} p<0.05,{ }^{* *} p<0.01$.

A

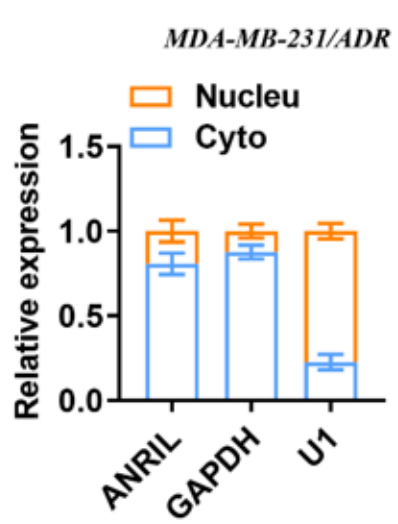

C

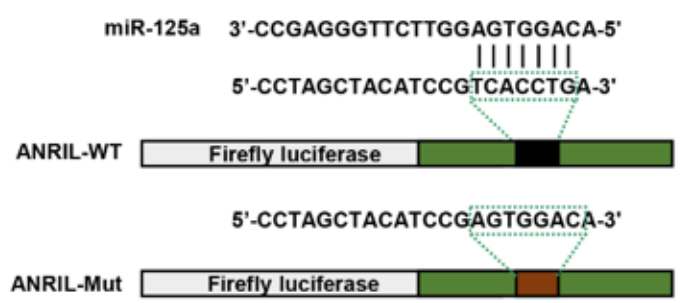

D

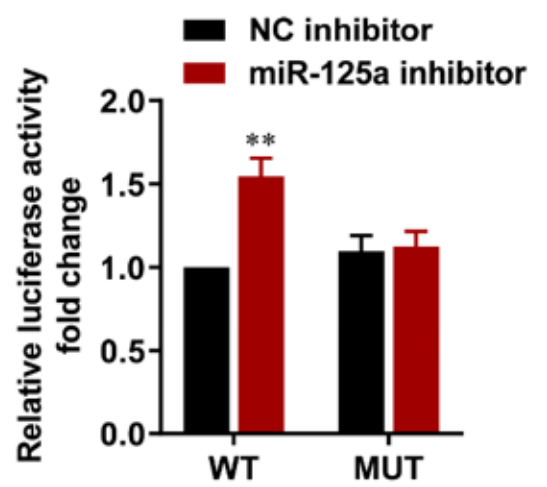

$\mathbf{F}$

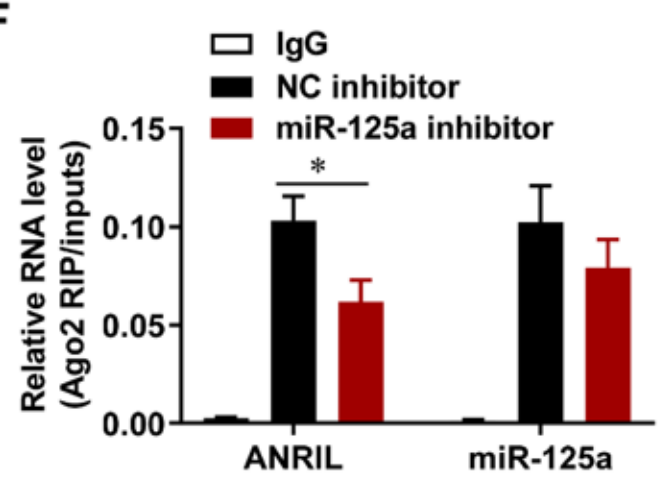

Figure 3

ANRIL directly binding to miR-125a in TNBC/ADR cells. (A) The subcellular location of ANRIL was determined by quantitative real-time PCR. (B) Sequence complementarity of ANRIL and miR-125a. Short vertical lines indicated complementary nucleotides. (C) The dual-luciferase reporter gene assay was

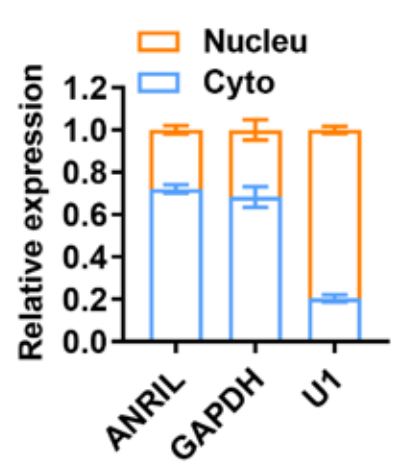

E

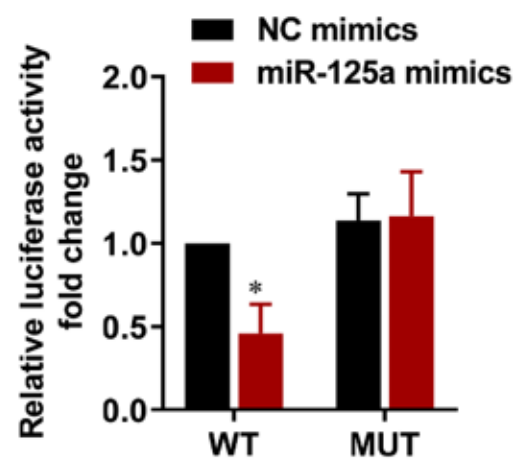


utilized in TNBC cells to verify the target relationship between ANRIL and miR-125a-mimic or miR-125ainhibitor (D). (E) The amount of ANRIL and miR-125a bound to Ago2 or IgG was measured by RT-qPCR in the presence of miR-125a inhibitor or negative control. Data represent the mean \pm SEM of three independent experiments. ${ }^{*} p<0.05,{ }^{* *} p<0.01$.

A

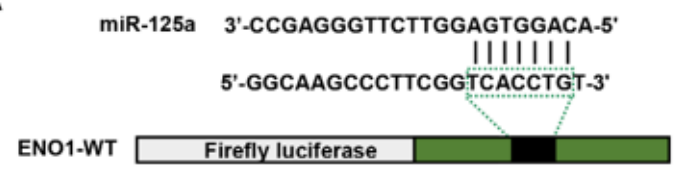

5'-GGCAAGCCCTTCGGAGTGGACT-3'

ENO1-Mut Firefly luciferase

B
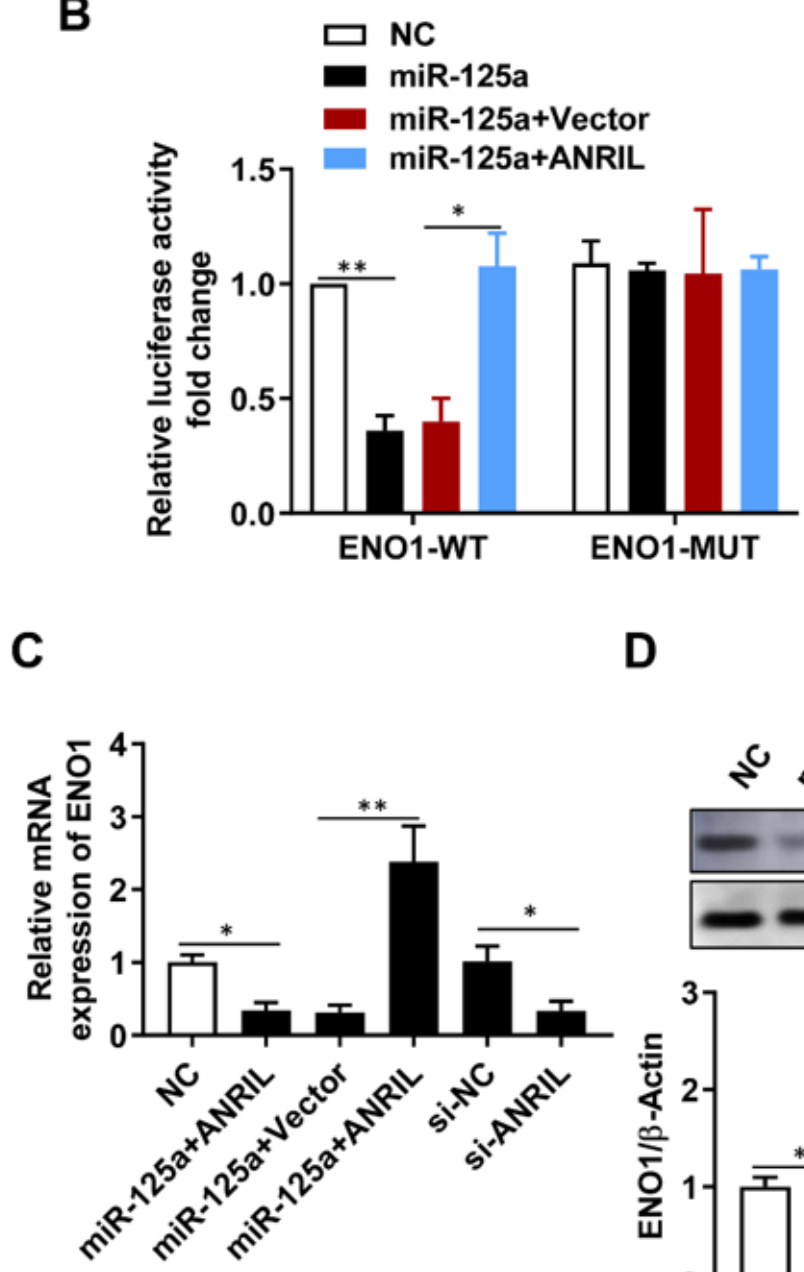

D
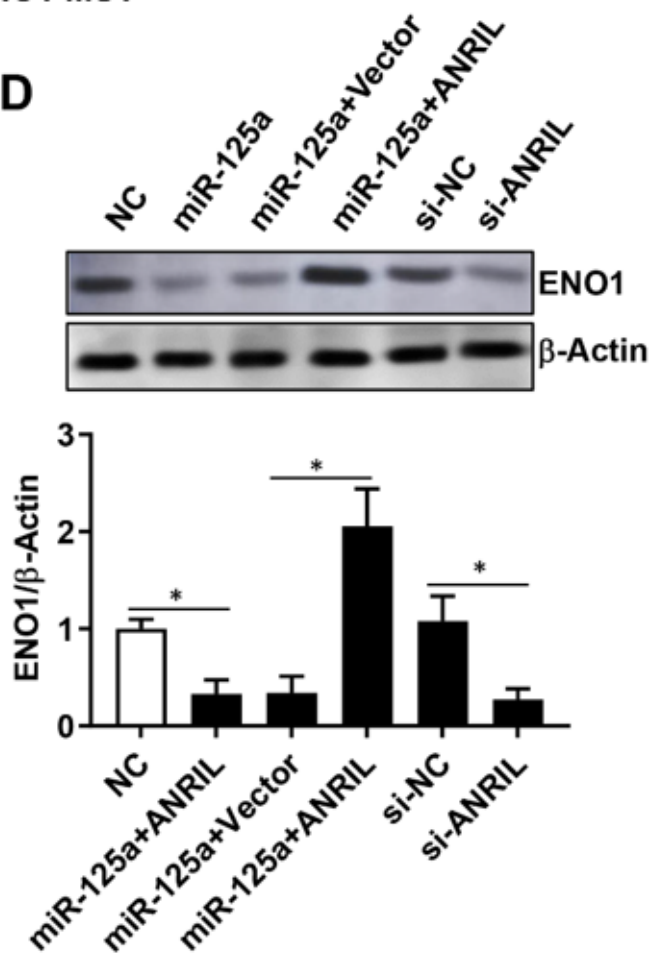

Figure 4 
ENO1 is a target of miR-125a and is positively regulated by ANRIL in TNBC cells. (A) Bioinformatics analysis using targeting algorithms showing that miR-125a had candidate binding sites in the 3'-UTR of EN01 mRNA. (B) Luciferase reporter assay implied that miR-125a overexpression remarkably inhibited the luciferase activities of ENO1-wt but not that of ENO1-mut in TNBC cells. (C) qRT-PCR result of ANRIL silencing and miR-125a overexpression reduced mRNA of ENO1 in TNBC cells. (D) Western blot analysis of ANRIL silencing and miR-125a overexpression reduced protein level of ENO1 in TNBC cells. ${ }^{\star} p<0.05$, $\star \star p p<0.01$

A

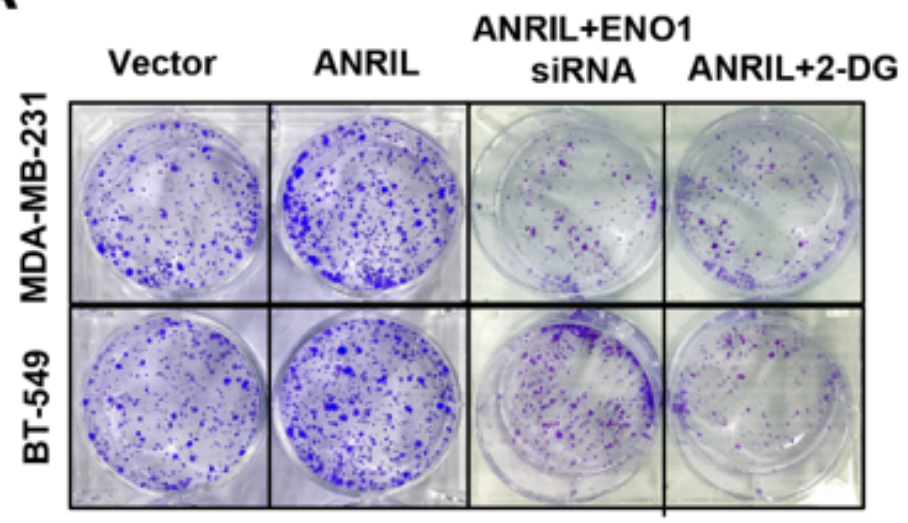

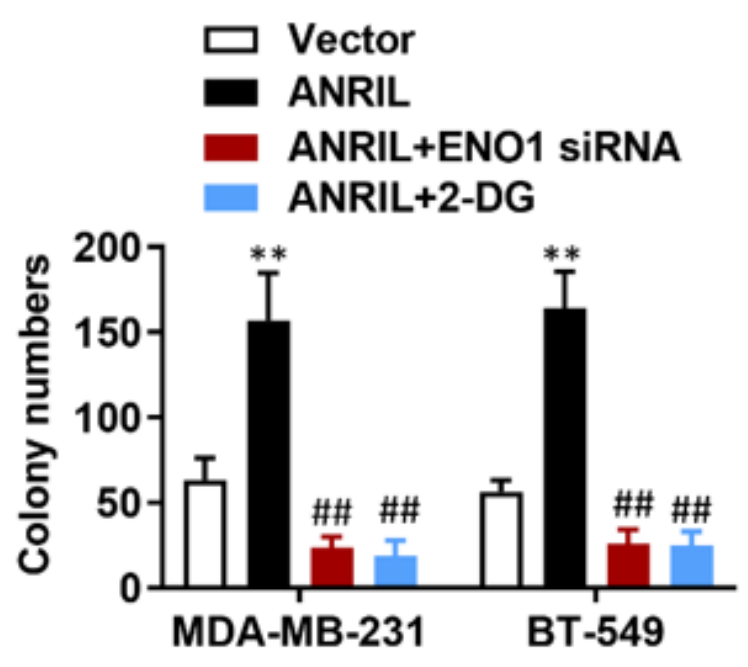

B

C

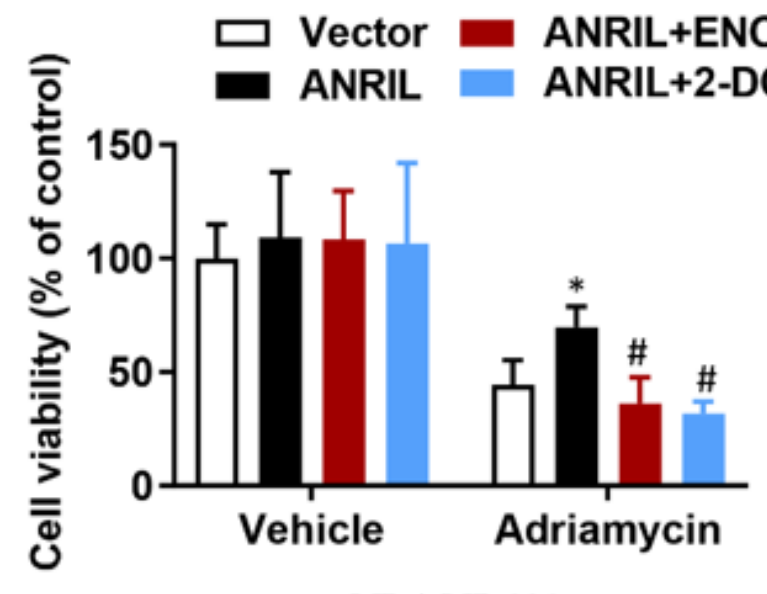

$M D A-M B-231$
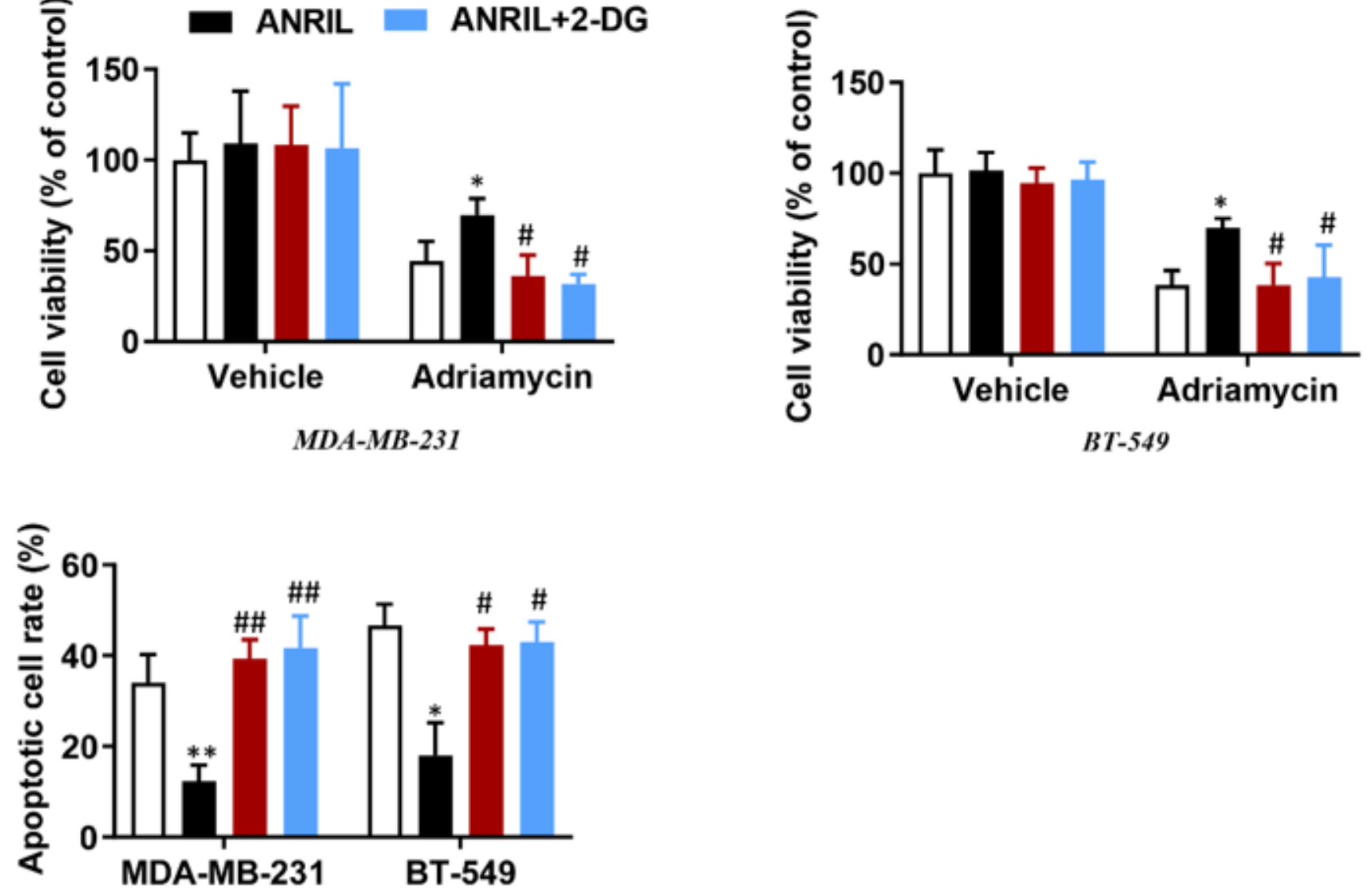
Figure 5

ANRIL increases TNBC cell chemoresistance via ENO1 in vitro. (A) ANRIL overexpression significantly enhanced the clonogenic potential of TNBC cells upon exposure to ADR. (B) Cell viability analysis showed that ANRIL enhanced ADR resistance of TNBC cells. (C) Flow cytometry analysis showed less apoptosis in ANRIL-overexpressing TNBC cells than in control cells treated with ADR.

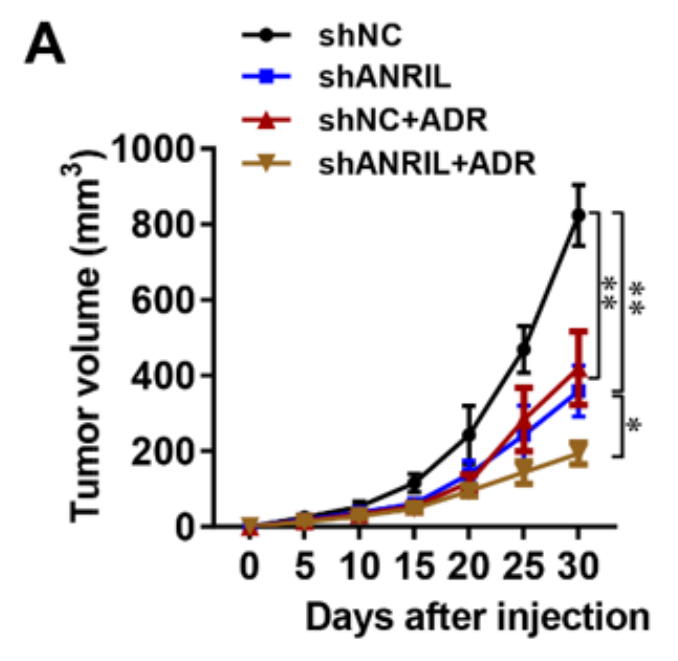

B

C
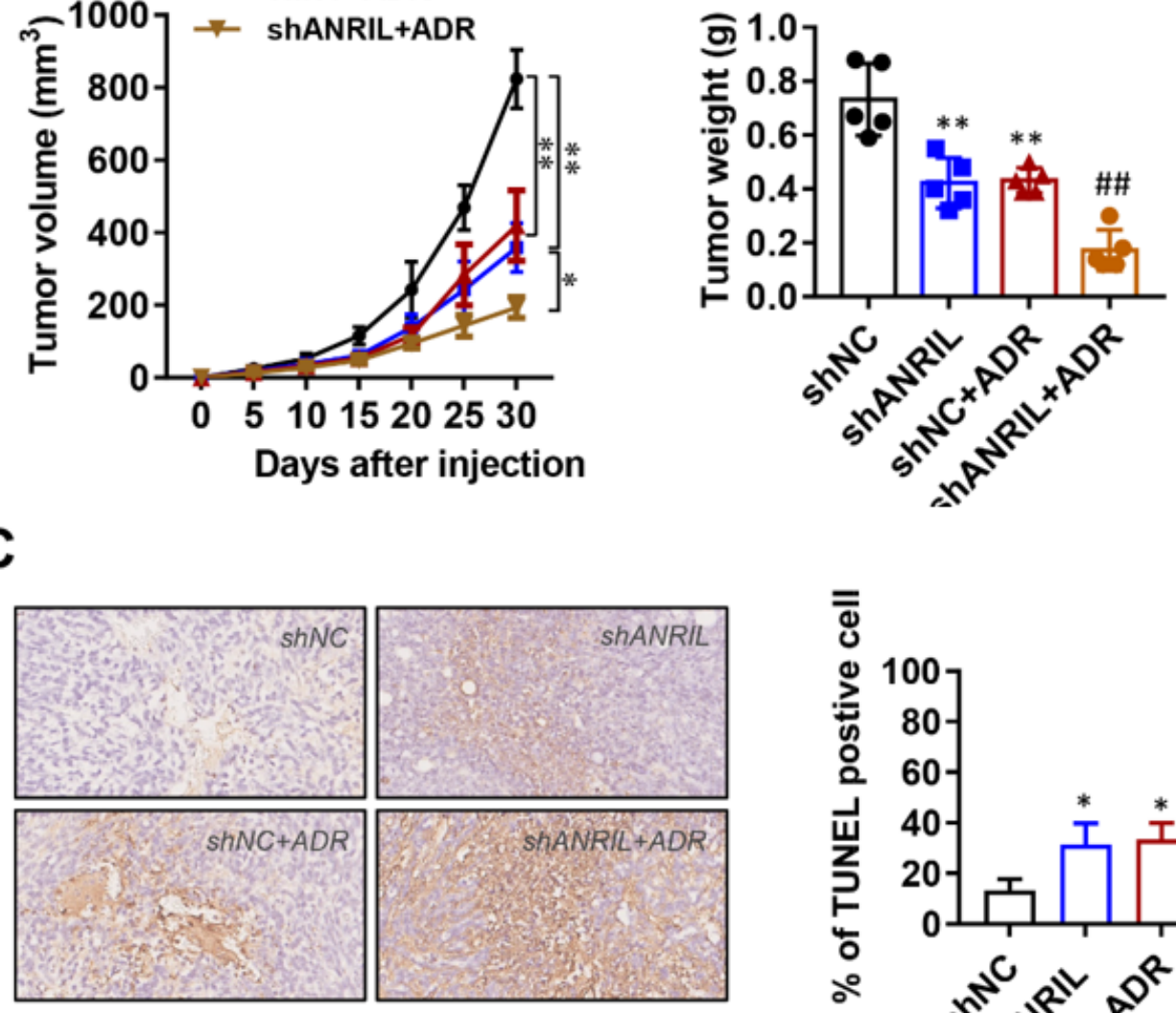

D
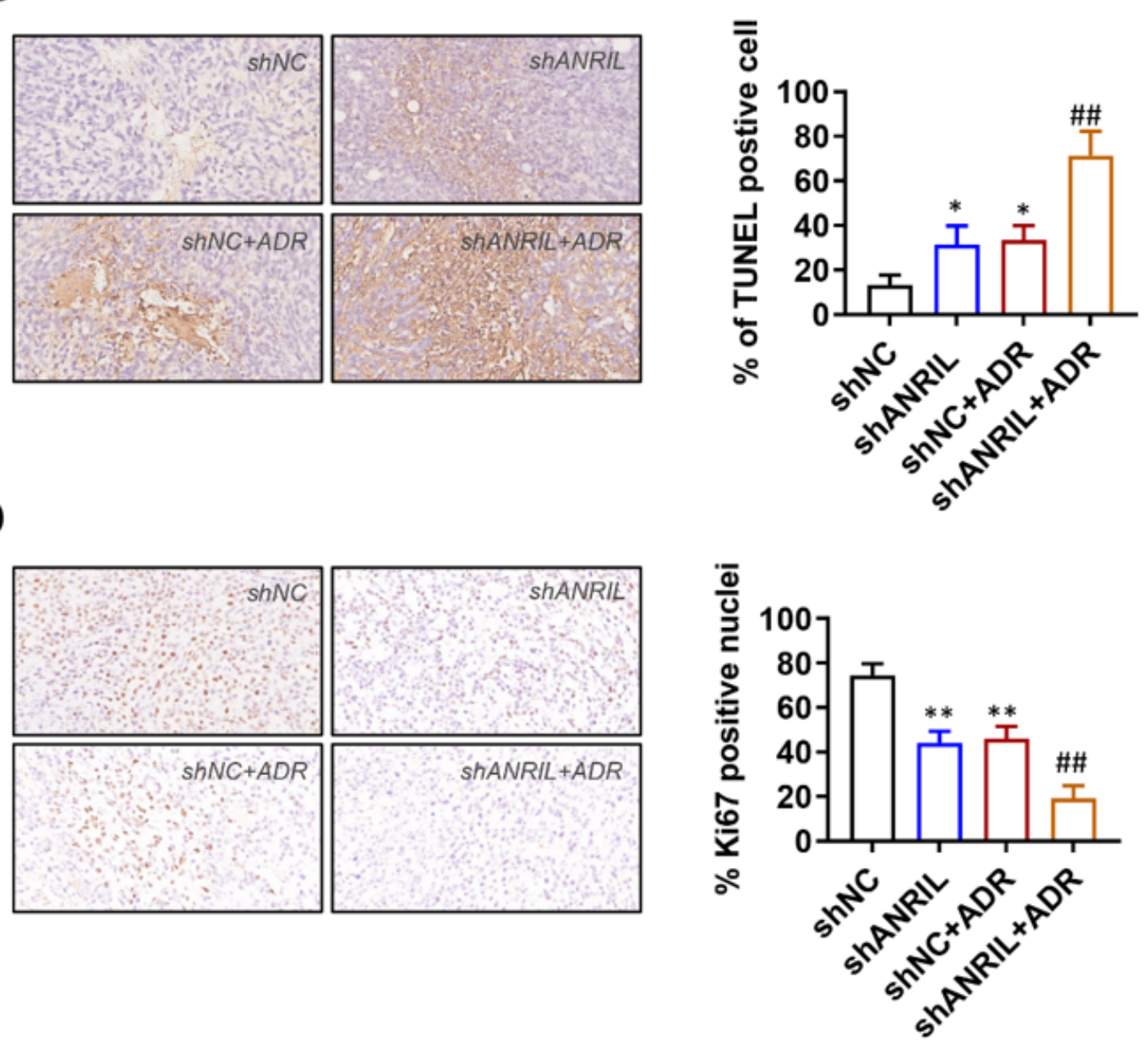

Figure 6 
ANRIL silencing inhibited tumor growth in vivo. (A) Quantitative analysis of the NB tumor volume at the indicated time. (B) Quantitative analysis of the NB tumor weight. (C, D) TUNEL assay (C) and immunohistochemistry of Ki-67 protein (D) in the TNBC tissues. TUNEL and Ki-67-positive cells indicate apoptotic and proliferative cells, respectively. ${ }^{\star} p<0.05,{ }^{*} \mathrm{p} p 0.01$.

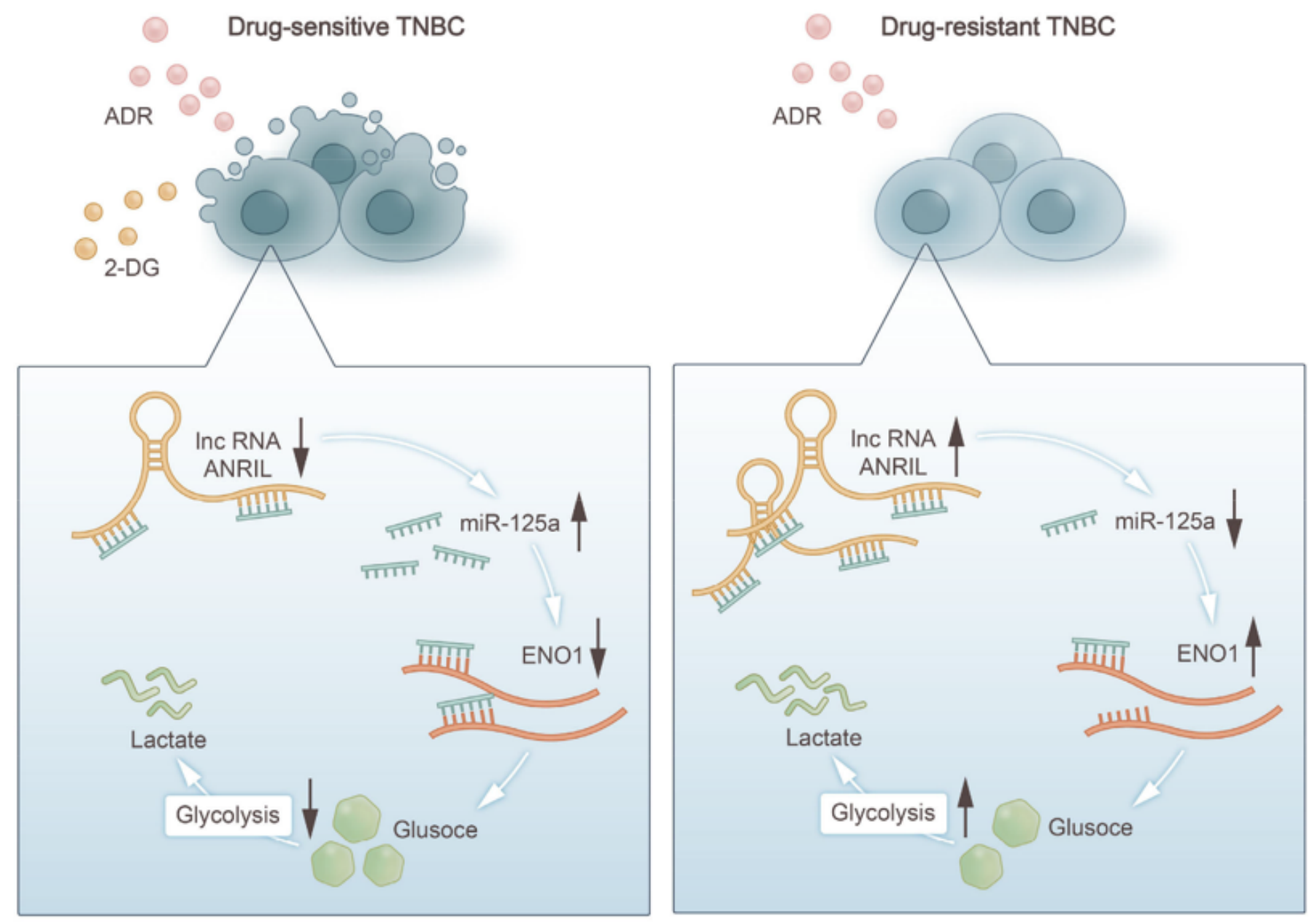

\section{Figure 7}

Schematic diagram of IncRNA-ANRIL-based regulatory mechanism in ADR resistance of TNBC cells. 